\title{
Development of systemic lupus erythematosus in an elderly male hemodialysis patient with pleuritis
}

\author{
Maki Tsukamoto $\cdot$ Kent Doi $\cdot$ Norio Hanafusa $\cdot$ \\ Miho Ohshima • Eisei Noiri - Kazuhiko Yamamoto • \\ Masaomi Nangaku
}

Received: 30 July 2012 / Accepted: 24 September 2012/Published online: 25 October 2012

(C) Japanese Society of Nephrology 2012

\begin{abstract}
This report describes a 70-year-old man who developed systemic lupus erythematosus (SLE) during treatment with maintenance hemodialysis. At initiation of maintenance dialysis, the etiology of end-stage renal disease (ESRD) was diabetic nephropathy and no clinical symptom or laboratory data suggested SLE. Fever, pleural effusion that did not respond to ultrafiltration, and immunological findings such as low complement and elevated anti-double-strand DNA antibody level appeared 4 years after maintenance dialysis initiation. Immunosuppressive therapy with corticosteroids improved these abnormalities remarkably. This case underscores the necessity of considering SLE in the differential diagnosis of pleural effusion with male ESRD patients, even if another etiology of ESRD exists.
\end{abstract}

Keywords Systemic lupus erythematosus - Elderly male · Hemodialysis · Pleuritis

\section{Introduction}

Systemic lupus erythematosus (SLE) is a chronic autoimmune disease characterized by multiple organ involvement,

M. Tsukamoto $\cdot$ K. Doi $\cdot$ N. Hanafusa $\cdot$ E. Noiri ·

M. Nangaku $(\bowtie)$

Department of Nephrology and Endocrinology,

Graduate School of Medicine, The University of Tokyo,

7-3-1 Hongo, Bunkyo-ku, Tokyo 113-8655, Japan

e-mail: mnangaku-tky@umin.ac.jp

M. Tsukamoto

e-mail: mtsukamoto-tky@umin.ac.jp

M. Ohshima $\cdot$ K. Yamamoto

Department of Allergy and Rheumatology, Graduate School

of Medicine, The University of Tokyo, Tokyo, Japan including renal failure. SLE commonly occurs in young and middle-aged females. It is widely recognized that SLE shows remission of disease activity, such as non-renal organ involvement and serological abnormalities after lupus nephropathy progresses to end-stage renal disease (ESRD), which requires renal replacement therapy [1]. This phenomenon is designated as burn-out lupus. However, some SLE patients reportedly showed reactivation or first presentation of lupus activity not only among females but also in young males [2-4]. No report in the relevant literature written in English has described a case of an elderly male hemodialysis patient who developed SLE after the initiation of hemodialysis.

\section{Case report}

A 70-year-old man developed ESRD and started maintenance hemodialysis 4 years ago. Although he has shown a stable clinical course since then, he suffered from remittent fever and fluctuating bilateral arthralgia of the knees for 4 weeks before he was transferred to our hospital for examination of fever of unknown etiology and pleural effusion that did not respond to reduced dry weight.

He had a medical history of hypertension and angina pectoris requiring coronary artery intervention. He was complicated with diabetes mellitus. Photocoagulation was conducted for diabetic retinopathy. Although renal biopsy was not performed, the etiology of ESRD in this case was regarded as diabetic nephropathy with hypertensive nephrosclerosis. No drug known to cause SLE had been prescribed.

Physical examination revealed bilaterally weakened respiratory sounds associated with mild chest pain. No lymphadenopathy or peripheral edema was found. 
Decreased blood oxygen concentration was detected with $\mathrm{SpO}_{2}$ of $80 \%$ in room air and his blood pressure was $106 / 56 \mathrm{mmHg}$, which was lower than that measured several months prior.

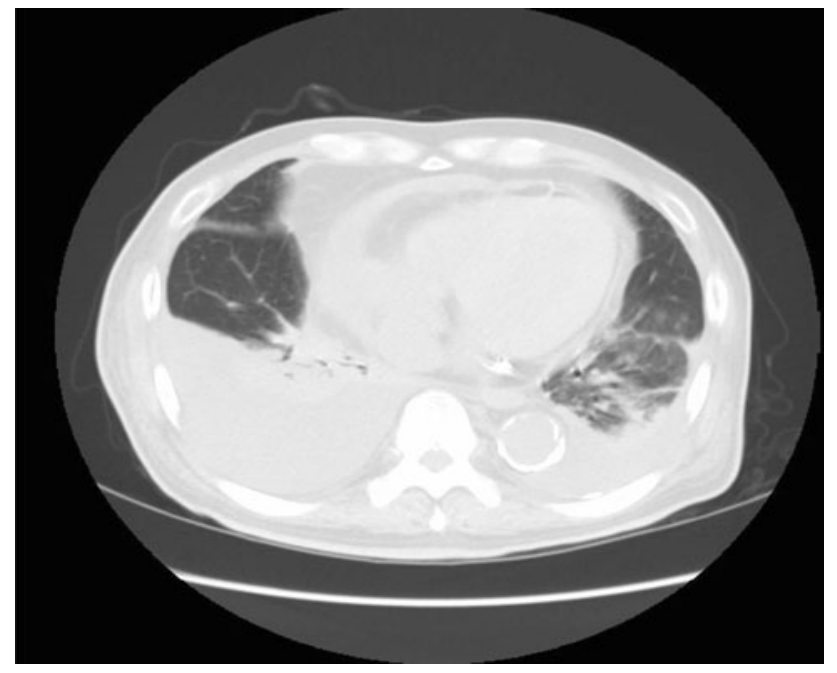

Fig. 1 Chest computed tomography (CT) showed pericardial and bilateral pleural effusion at transfer to our hospital
Laboratory findings were as follows: white blood cells (WBC) $5500 / \mu \mathrm{L}$ (Seg 71.2 \%, Mono 4.6\%, Lym $18.9 \%$ ), $\mathrm{Hb} \quad 8.3 \mathrm{~g} / \mathrm{dL}$, platelet $284000 / \mu \mathrm{L}$, C-reactive protein $13.78 \mathrm{mg} / \mathrm{dL}$, positive for anti-nuclear antibody $(320 \times$, homogeneous pattern), anti-ds DNA antibody of $45 \mathrm{IU} /$ $\mathrm{mL}, \mathrm{C} 369 \mathrm{mg} / \mathrm{dL}, \mathrm{C} 412 \mathrm{mg} / \mathrm{dL}, \mathrm{CH} 5032.6 \mathrm{U} / \mathrm{mL}$, IC $4.4 \mu \mathrm{g} / \mathrm{mL}$. There was no presence of hemolytic anemia, negative for anti-Sm antibody, LAC, anti-cardiolipin antibody, rheumatoid factor, anti-RNP antibody, P-ANCA, and C-ANCA. Plain chest computed tomography (CT) showed pericardial and bilateral pleural effusion (Fig. 1). Upper and lower gastrointestinal tract endoscopies showed no evidence of malignancy. No viral or bacterial infection, including tuberculosis, was detected by appropriate tests.

Thoracocentesis was conducted to diagnose the cause of pleural effusion. Clear yellow exudative pleural effusion was obtained. The results of laboratory tests on the pleural effusion were as follows: WBC 700/ $\mu \mathrm{L}$ (Seg $62.0 \%$, Mono $11.0 \%$, Lym $26 \%$ ), total protein $4.2 \mathrm{~g} / \mathrm{dL}$ (ratio to serum 0.70), LDH $312 \mathrm{IU} / \mathrm{dL}$ (ratio to serum 0.66), glucose $118 \mathrm{mg} / \mathrm{dL}$ (ratio to serum 0.80), anti-nuclear antibody (320 $\times$, homogeneous pattern), anti-ds DNA antibody of $24 \mathrm{IU} / \mathrm{mL}, \mathrm{C} 326 \mathrm{mg} / \mathrm{dL}, \mathrm{C} 42 \mathrm{mg} / \mathrm{dL}$. Bacterial culture
Fig. 2 Chest X-ray showed disappearance of bilateral pleural effusion after steroid therapy
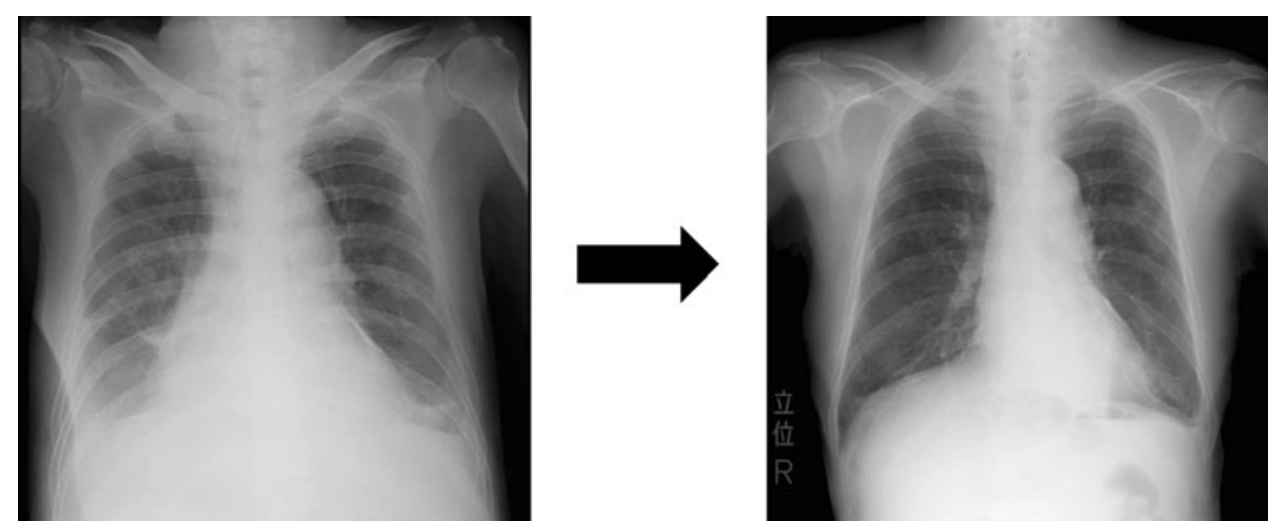

Fig. 3 Clinical course of anti-ds DNA antibody and complement with steroid therapy

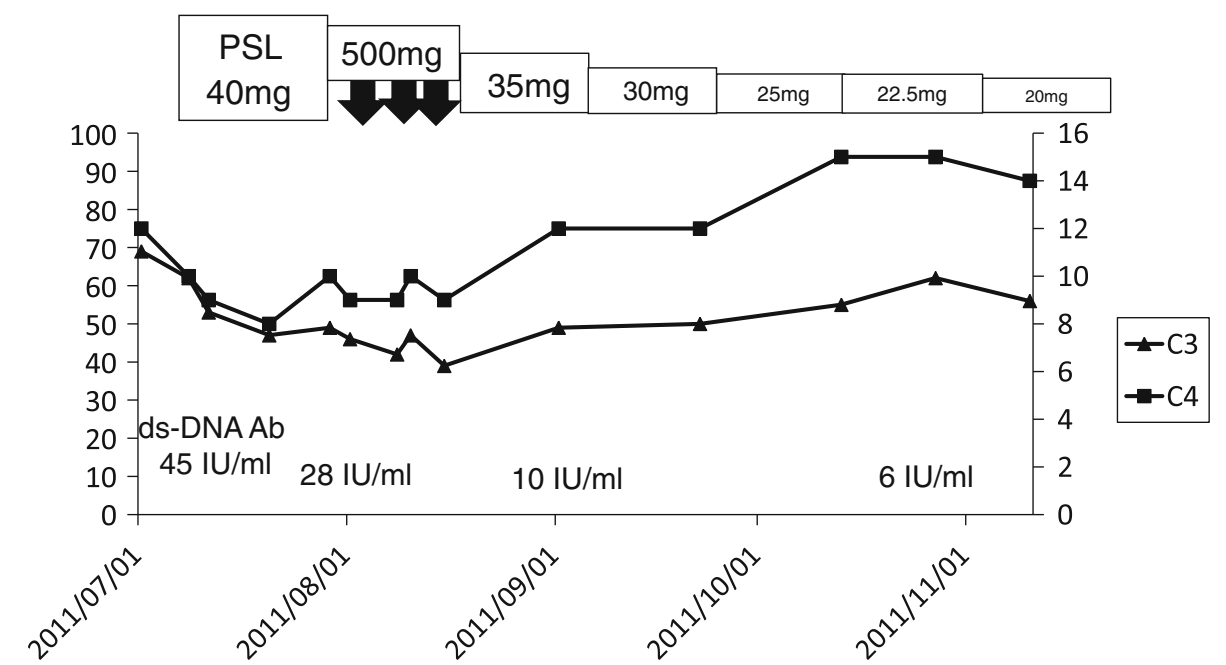


and polymerase chain reaction (PCR) for tuberculosis were negative. Cytology tests were negative for any malignancy.

This case showed 5 of 11 symptoms among the criteria established by the American College of Rheumatology (ACR) (serositis, arthritis, lymphocytopenia, positive antinuclear, and anti-ds DNA antibodies) Therefore, he was diagnosed as SLE. Oral prednisolone at the dose of $40 \mathrm{mg} /$ day $(0.8 \mathrm{mg} / \mathrm{kg} /$ day $)$ remarkably improved fever and arthritis. Anti-ds DNA antibody titer was promptly normalized, although hypocomplementemia responded later (Fig. 2). After steroid therapy, chest X-ray revealed complete disappearance of pleural effusion, with no change of dry weight of maintenance dialysis (Fig. 3).

\section{Discussion}

SLE was originally reported in the nineteenth century as a skin disease, but it is now recognized as a chronic autoimmune disease characterized by multiple organ involvement [5]. Because of the clinical heterogeneity of the disease, the diagnosis of SLE requires at least 4 of the 11 symptoms determined by the ACR [6]. As described herein, three features make it difficult to diagnose SLE: gender, age, and ESRD.

As with many other autoimmune diseases, SLE affects females more frequently than males. Reportedly, 4-22\% of SLE occurs in males [7]. Male SLE patients are more likely to have end organ damage, including neuropsychiatric, renal, cardiovascular, peripheral vascular disease, and myocardial infarction. Therefore, males have higher mortality [7]. However, the precise reason for why male SLE appears to be more severe is unknown [8].

Although SLE usually occurs in younger patients, it developed in this case at the age of 74 years. The incidence of late-onset SLE (beginning after 50-65 years of age) is reportedly 12-18\% [9]. Late-onset SLE patients appear to be present with serositis, lung involvement, and Sjogren's syndrome more often than with skin manifestations, photosensitivity, or nephritis [9]. Serologically, a higher rate of positive rheumatoid factor and negative anti-RNP antibodies is observed in late-onset SLE. In addition, hypocomplementemia is rarely found in elderly SLE patients [9]. The present case showed serositis and mild hypocomplementemia.

Systemic lupus erythematosus can achieve a remission of active inflammation ("burn out"), especially after patients develop advanced renal failure, which necessitates dialysis therapy [10]. Typically, young SLE female patients with lupus nephritis show no blood test abnormality several years after dialysis initiation. However, SLE can occur in ESRD patients. Al-Hawas et al. [4] reported a 38-year-old male dialysis patient who developed SLE. He showed fever, lethargy, body aches, and pancytopenia after 14 years of hemodialysis. No report in the literature describes SLE occurrence in elderly ESRD male patients.

Infection and malignancy are the most likely causes of fever accompanied with pleural effusion, especially in elderly patients. In addition, fluid overload is considered when a dialysis patient shows bilateral pleural effusion. The present case underscores the necessity of adding SLE to the differential diagnosis list in elderly male dialysis patients with pleural effusion because early diagnosis of SLE can prevent the progression of organ impairment and a fatal event.

Conflict of interest None declared.

\section{References}

1. Jarrett MP, Santhanam S, Del Greco F. The clinical course of end-stage renal disease in systemic lupus erythematosus. Arch Intern Med. 1983;143:1353-6.

2. Friedman AS, Folkert V, Khan GA. Recurrence of systemic lupus erythematosus in a hemodialysis patient presenting as a unilateral abducens nerve palsy. Clin Nephrol. 1995;44:338-9.

3. Hernandez-Jaras J, Bernis C, Paraiso V, Barril G, Alvarez V, Traver JA. Development of systemic lupus erythematosus in a patient on hemodialysis. Am J Nephrol. 1992;12:105-7.

4. Al-Hawas F, Abdalla AH, Al-Sulaiman MH, Mousa DH, Owda AK, Al-Khader AA. Development of systemic lupus erythematosus in a male patient after 14 years on hemodialysis. Am J Kidney Dis. 1997;29:631-2.

5. Tsokos GC. Systemic lupus erythematosus. N Eng J Med. 2011;365:2110-21.

6. Tan EM, Cohen AS, Fries JF, et al. The 1982 revised criteria for the classification of systemic lupus erythematosus. Arthritis Rheum. 1982;25:1271-7.

7. Tan TC, Fang H, Magder LS, Petri MA. Differences between male and female systemic lupus erythematosus in a multiethnic population. J Rheumatol. 2012;39:759-69.

8. de Carvalho JF, do Nascimento AP, Testagrossa LA, Barros RT, Bonfá E. Male gender results in more severe lupus nephritis. Rheumatol Int. 2010;30:1311-5.

9. Rovenský J, Tuchynová A. Systemic lupus erythematosus in the elderly. Autoimmun Rev. 2008;7:235-9.

10. Coplon NS, Diskin CJ, Petersen J, Swenson RS. The long-term clinical course of systemic lupus erythematosus in end-stage renal disease. N Engl J Med. 1983;308:186-90. 